\title{
Role of ${ }^{28} \mathrm{Si}$ excitations in the sub-barrier fusion of ${ }^{28} \mathrm{Si}+{ }^{120} \mathrm{Sn}$
}

\author{
Lagy T. Baby, * Vandana Tripathi, J. J. Das, P. Sugathan, N. Madhavan, and A. K. Sinha \\ Nuclear Science Centre, New Delhi 110 067, India
}

M. C. Radhakrishna

Department of Physics, Bangalore University, Bangalore 560 056, India

P. V. Madhusudhana Rao

Department of Nuclear Physics, Andhra University, Visakhapatnam 530 003, India

\author{
S. K. Hui \\ Department of Physics, Calcutta University, Calcutta 700 075, India
}

K. Hagino

Institute for Nuclear Theory, Department of Physics, University of Washington, Seattle, Washington 98195

(Received 1 September 1999; published 31 May 2000)

\begin{abstract}
Fusion cross sections were measured for the systems ${ }^{16} \mathrm{O}$ and ${ }^{28} \mathrm{Si}+{ }^{120} \mathrm{Sn}$ in order to understand the role of specific channels involved in sub-barrier fusion. Fusion cross sections were measured by direct detection of the evaporation residues. Extracted fusion barrier distributions were analyzed using the exact coupled-channel code CCFULL as well as the simplified code CCMOD. These calculations show that the observed fusion barrier distribution for the $\mathrm{Si}+\mathrm{Sn}$ system is reproduced only if the rotational excitation in ${ }^{28} \mathrm{Si}$ is taken into account.
\end{abstract}

PACS number(s): 25.70.Jj, 24.10.Eq

\section{INTRODUCTION}

Recent interest in low-energy heavy ion reactions has been focused towards an understanding of the reaction mechanism in the framework of the coupled-channel formalism. A complete understanding of the role of various reaction channels in enhancing the sub-barrier fusion cross section has not yet been achieved, even though in some systems the effect of coupling to a few specific channels has been identified. With the method of exploiting high-precision fusion data to extract the barrier distribution experimentally $[1,2]$, it has now become possible to test the finer details of the channel coupling approach. A few measurements have been done in this direction where the barrier distribution has been extracted from the second derivative of the product of $\sigma_{f u s}$ and energy with respect to energy. These measurements have shown that coupling to several channels such as complex surface vibrations [3], few nucleon transfer channels [4], and static deformations [5,6] stands out clearly when the cross section is represented in the form of a barrier distribution.

The ground state deformation of the colliding partners has been found to influence dramatically the sub-barrier fusion cross sections. In this case, the different orientations of the deformed target with respect to the beam direction give rise to a distribution of barriers. This results in considerable en-

\footnotetext{
*Affiliation: Department of Physics, Bangalore University, Bangalore 560 056, India; electronic address: lagy@tifr.res.in; present address: Department of Nuclear and Atomic Physics, Tata Institute of Fundamental Research, Mumbai 400 005, India.
}

hancement of fusion cross sections at sub-barrier energies. Note that this picture relies on an assumption that the orientation remains constant (frozen) during the collision process. This approximation works well for heavy nuclei where the excitation energies of the rotational band are small. The experimental fusion barrier distributions were analyzed in this manner in Refs. [5,6]. For light deformed nuclei, however, the frozen approximation may not be valid [7] because they have usually high-lying rotational states and thus neglecting the excitation energy is not reasonable.

In order to study the role of light deformed nuclei in the fusion mechanism, we carried out fusion and barrier distribution measurements for the systems ${ }^{16} \mathrm{O},{ }^{28} \mathrm{Si}+{ }^{120} \mathrm{Sn}$. The ${ }^{28} \mathrm{Si}$ nucleus has a large ground state quadrupole deformation of $\beta_{2}=-0.4$ and hexadecapole deformation $\left(\beta_{4}=0.1\right)$, and thus provides an ideal case for our purpose. The doubly magic ${ }^{16} \mathrm{O}$ projectile was used as a comparison, which has high-lying vibrational states. Since ${ }^{16} \mathrm{O}$ behaves as an inert nucleus in fusion, the channel coupling effects in the ${ }^{120} \mathrm{Sn}$ target can be assessed through the ${ }^{16} \mathrm{O}+{ }^{120} \mathrm{Sn}$ measurement.

This paper is organized as follows. Section II gives the details of the experimental technique and data reduction. Section III explains the coupled-channel calculations employed to analyze the data and the last section gives a summary.

\section{EXPERIMENTAL DETAILS}

The experiment was performed using the ${ }^{16} \mathrm{O}$ and ${ }^{28} \mathrm{Si}$ beams provided by the 15UD Pelletron at the Nuclear Science Center (NSC), New Delhi. The target used was an iso- 


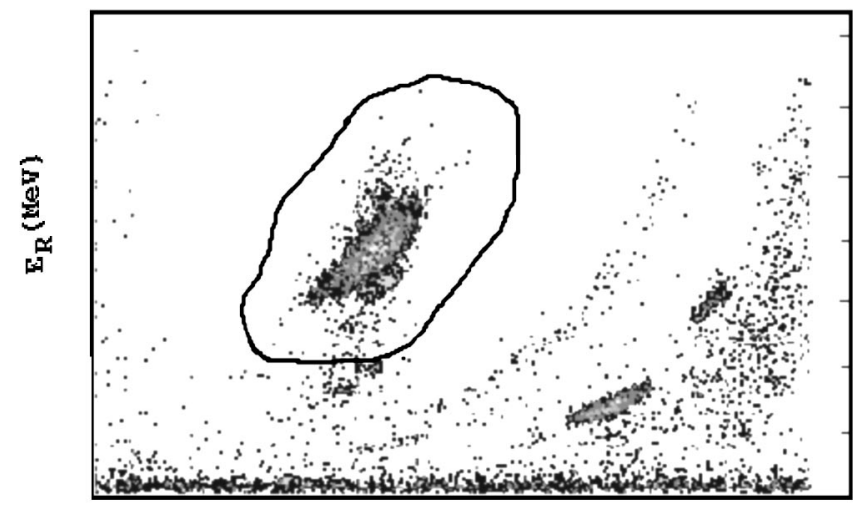

TOF (Arb. Units)

FIG. 1. A two-dimensional plot for ${ }^{28} \mathrm{Si}+{ }^{120} \mathrm{Sn}$ at a beam energy of $115 \mathrm{MeV}$. The $X$ axis is the time of flight and $Y$ axis is the total energy of the residues. The marked area represents the evaporation residues.

topically enriched (98\%) $50 \mu \mathrm{g} / \mathrm{cm}^{2}{ }^{120} \mathrm{Sn}$ foil with a $15 \mu \mathrm{g} / \mathrm{cm}^{2}$ carbon backing. In these measurements we have used pulsed beams of ${ }^{16} \mathrm{O}$ and ${ }^{28} \mathrm{Si}$ with repetition rates of $4 \mu \mathrm{sec}$ and $2 \mu \mathrm{sec}$, respectively. Fusion excitation function measurements were carried out at laboratory energies from 50 to $68 \mathrm{MeV}$ in $0.5 \mathrm{MeV}$ steps for the $\mathrm{O}+\mathrm{Sn}$ system and from 95 to $125 \mathrm{MeV}$ in $0.75-1 \mathrm{MeV}$ steps for the $\mathrm{Si}+\mathrm{Sn}$ system. The experiment was performed using the recoil mass separator HIRA (Heavy Ion Reaction Analyzer) [8] at NSC. The evaporation residues were focused at the focal plane of HIRA after dispersing them according to their $\mathrm{m} / \mathrm{q}$ values. A position sensitive $50 \mathrm{~mm} \times 50 \mathrm{~mm}$ silicon strip detector was used to detect the evaporation residues (ERs) at the focal plane of HIRA. The flight time of residues through HIRA were around $3 \mu \mathrm{sec}$ and $1.5 \mu \mathrm{sec}$, respectively, for the two cases. A time of flight between the rf signal and focal plane timing along with the total energy of the residues was helpful in getting a very good suppression of the background. A twodimensional spectrum of time of flight vs total energy of the ER is shown in Fig. 1 where the good separation between the ERs and background is clearly seen. Four silicon surface barrier detectors were used for exact monitoring of the beam and also for normalization. These detectors were kept at an out of plane angle of $20^{\circ}$ with respect to the beam direction, giving a reaction angle of $28^{\circ}$. At this forward angle the elastic cross section was taken to be fully Rutherford. A thin carbon foil of $\approx 5 \mu \mathrm{g} / \mathrm{cm}^{2}$ was inserted at a distance of $\sim 10 \mathrm{~cm}$ away from the target in the direction of the residues so that the charge state of the residue shifted due to internal conversion is re-equilibrated. For the measurement of evaporation residue cross section, the HIRA solid angle was set at 5 msr. The ER cross section was measured with HIRA at $0^{\circ}$ with respect to the beam direction.

In the measurement of the fusion cross section, the evaporation residue cross section was taken to be equal to the total fusion cross section since the fission contribution in this energy region is negligible. The residue cross section was obtained from the measured ER yield using the expression

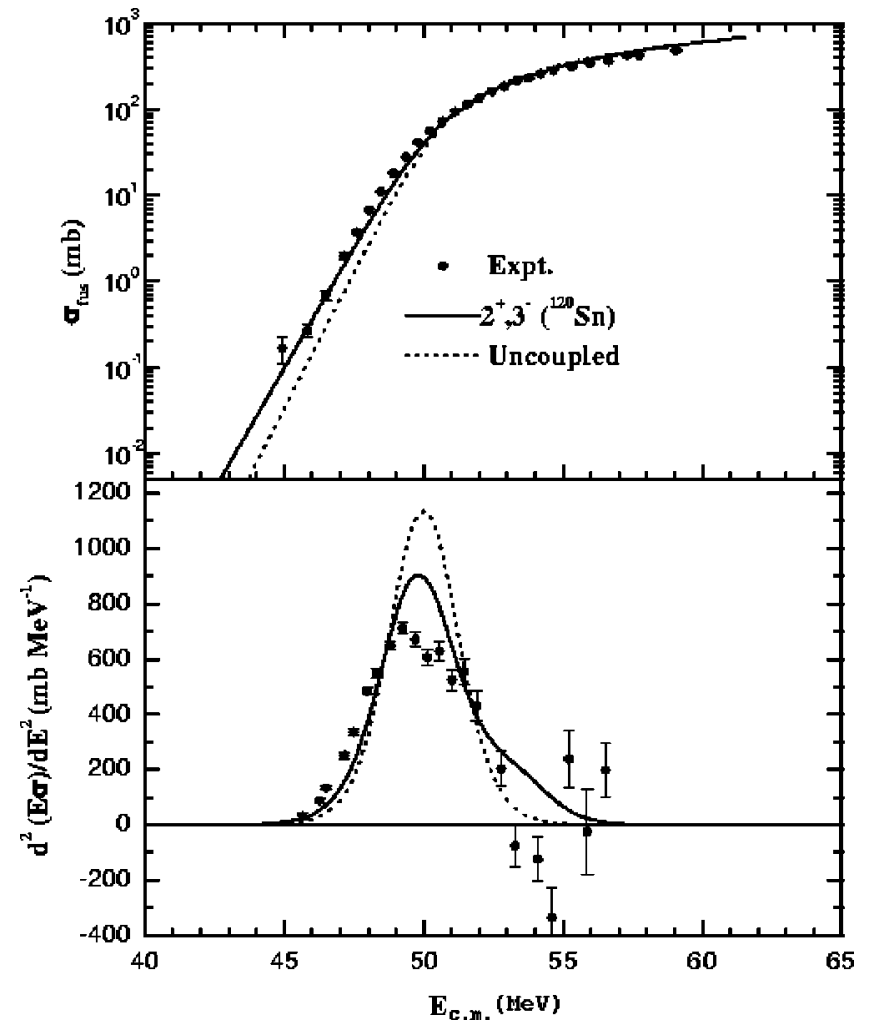

FIG. 2. Fusion excitation function (top) and barrier distribution (bottom) for ${ }^{16} \mathrm{O}+{ }^{120} \mathrm{Sn}$. The results of the simplified coupledchannel calculations are also shown (solid line). The dotted line gives the prediction of the one-dimentional barrier penetration model.

$$
\sigma_{f u s}=\frac{1}{\epsilon}\left(\frac{Y_{R}}{Y_{M}}\right)\left(\frac{d \sigma}{d \Omega}\right)_{R} \Omega_{M},
$$

where $\epsilon$ is the average HIRA efficiency, $Y_{R}$ is the yield of the evaporation residues, $Y_{M}$ is the monitor counts, $(d \sigma / d \Omega)_{R}$ is the Rutherford cross section in the laboratory system, and $\Omega_{M}$ is the solid angle subtended by the monitor detector. The error in the measured cross section includes the uncertainty in the estimated HIRA efficiency which is around $10 \%$. It has been shown that the measured efficiency of the rms agrees well with the calculated efficiency $[9,10]$ from the PACE [11] distributions. Hence the average HIRA efficiency $\epsilon$ used in Eq. (1) was estimated from the measured charge state and energy distributions of the residues along with the energy and angular distribution predicted by the Monte Carlo code PACE. The efficiency of HIRA for the $\mathrm{Si}+\mathrm{Sn}$ system was $6.5 \%$ and that for the $\mathrm{O}+\mathrm{Sn}$ system was $3.5 \%$. Variation in the $\epsilon$ over the range of beam energies considered was found to be very small and has been therefore treated as energy independent. The barrier distribution was extracted from the fusion excitation function as the second derivative of $E \sigma_{f u s}$ with respect to the energy [1]. The second derivative was calculated using a point difference method as given below. The barrier distribution is defined at energy $\left(E_{1}+2 E_{2}+E_{3}\right) / 4$ as 
TABLE I. The deformation parameters, excitation energies, and the multipolarities of the states of different nuclei used in the coupled-channel calculations.

\begin{tabular}{llcc}
\hline \hline Nuclei & $J^{\pi}$ & $E_{x}(\mathrm{MeV})$ & $\beta$ \\
\hline${ }^{28} \mathrm{Si}$ & $2^{+}$ & 1.78 & -0.407 \\
& $4^{+}$ & 4.67 & \\
${ }^{120} \mathrm{Sn}$ & $2^{+}$ & 1.17 & 0.107 \\
& $3^{-}$ & 2.40 & 0.15 \\
\hline \hline
\end{tabular}

$$
\begin{aligned}
\frac{d^{2}\left(E \sigma_{f u s}\right)}{d E^{2}}= & 2\left(\frac{\left(E \sigma_{f u s}\right)_{3}-\left(E \sigma_{f u s}\right)_{2}}{E_{3}-E_{2}}\right. \\
& \left.-\frac{\left(E \sigma_{f u s}\right)_{2}-\left(E \sigma_{f u s}\right)_{1}}{E_{2}-E_{1}}\right)\left(\frac{1}{E_{3}-E_{1}}\right),
\end{aligned}
$$

where $\left(E \sigma_{f u s}\right)_{i}$ are evaluated at energies $E_{i}$. A $\Delta E$ of 2 $\mathrm{MeV}$ (lab) was used for the oxygen system and $2.5 \mathrm{MeV}$ (lab) for the silicon system to obtain the second derivative. Here $\Delta E$ is the energy step taken for extracting the second derivative. The statistical error $\delta_{c}$ associated with the second derivative at energy $E$ was calculated using the equation

$$
\delta_{c}=\left(\frac{E}{\Delta E^{2}}\right)\left[\left(\delta \sigma_{f u s}\right)_{1}^{2}+4\left(\delta \sigma_{f u s}\right)_{2}^{2}+\left(\delta \sigma_{f u s}\right)_{3}^{2}\right]^{1 / 2},
$$

where $\left(\delta \sigma_{f u s}\right)_{i}$ are the absolute errors in the cross sections. Since $\delta_{c}$ is proportional to the value of $\sigma_{f u s}$, for cross sections measured with a fixed percentage error, the barrier distribution becomes less defined at higher energies where the cross sections are high.

The measured cross sections and the barrier distribution for the $\mathrm{O}+\mathrm{Sn}$ system and $\mathrm{Si}+\mathrm{Sn}$ system are shown in Fig. 2 (see also Fig. 4, below). As seen in the figure, the barrier distribution for $\mathrm{O}+\mathrm{Sn}$ system shows a broad single peak with some fluctuations seen at the highest energies. In the case of $\mathrm{Si}+\mathrm{Sn}$, the barrier distribution shows a multiple peak structure with larger fluctuations at the highest energies in comparison to the $\mathrm{O}+\mathrm{Sn}$ case. The negative values seen in the barrier distribution around $90 \mathrm{MeV}$ could be due to an error in the measured cross section which was not accounted for in the present case.

\section{RESULTS AND ANALYSIS}

We employed the coupled-channel formalism to analyze the data. Simplified coupled-channel calculations [12,13] were first performed for the ${ }^{16} \mathrm{O}+{ }^{120} \mathrm{Sn}$ reaction using a modified version of the code CCMOD [14]. Here, the fusion

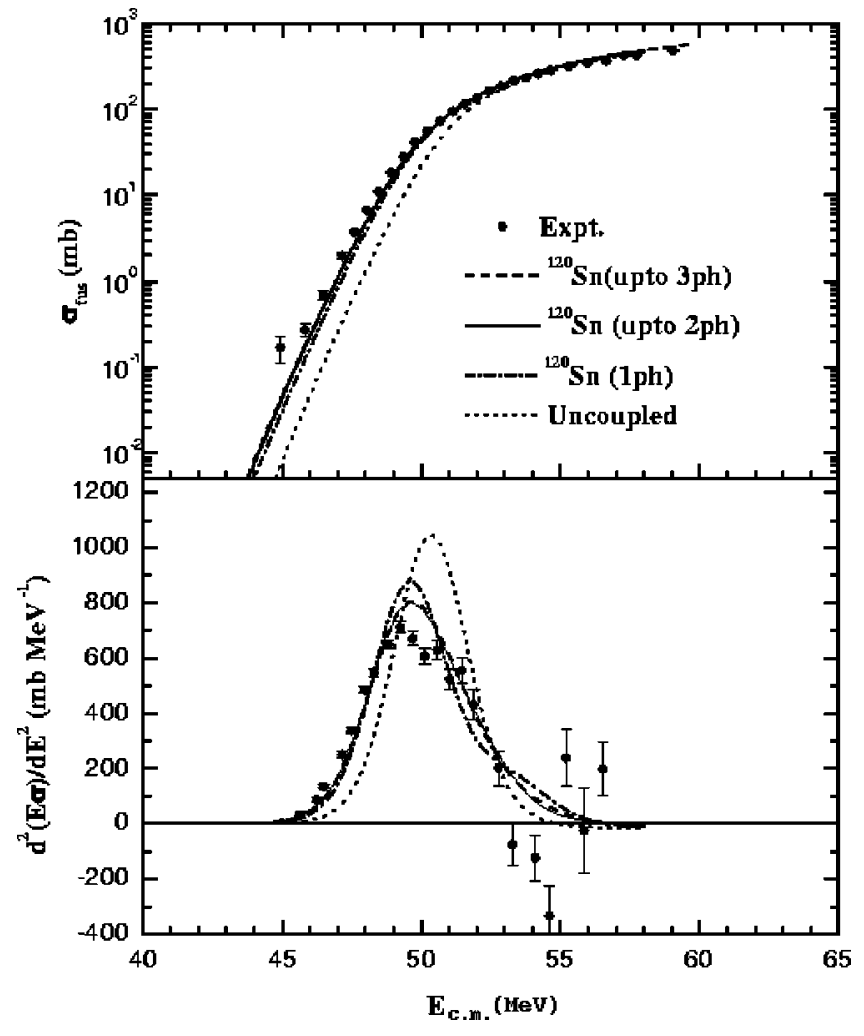

FIG. 3. Fusion excitation function and barrier distribution for ${ }^{16} \mathrm{O}+{ }^{120} \mathrm{Sn}$. The results of the exact coupled-channel calculations with coupling up to one-phonon, two-phonon, and three-phonon states of Sn are shown. The results of the two-phonon and threephonon couplings are almost overlapping.

cross section is calculated assuming the Hill-Wheeler [15] form for the transmission coefficient through a parabolic barrier. The coupled Schrödinger equations are solved using the following approximations. First, the finite excitation energy of the internal degrees of freedom is ignored. In the second approximation, the relative and intrinsic parts in the coupling interaction are separated in the adiabatic limit, where it is assumed that the intrinsic structure of the nuclei is not disturbed by the relative motion. Having done this, the coupling matrix is diagonalized and the weight factors for each of the new barriers are determined at the position of the uncoupled barrier. The inelastic coupling strengths are calculated using

$$
F_{\text {inel }}(r)=\frac{\beta_{\lambda}}{\sqrt{4 \pi}}\left[-R \frac{d V_{n}(r)}{d r}+\frac{3 Z_{1} Z_{2} e^{2}}{(2 \lambda+1)} \frac{R^{\lambda}}{r^{\lambda+1}}\right]
$$

where $\beta_{\lambda}$ is the deformation parameter with multipolarity $\lambda$. The first term gives the excitations due to nuclear part and

TABLE II. The potential parameters for the two systems used in the coupled-channel calculations.

\begin{tabular}{ccccccc}
\hline \hline System & $V_{b}(\mathrm{MeV})$ & $R_{b}(\mathrm{fm})$ & $\hbar \omega(\mathrm{MeV})$ & $a_{0}(\mathrm{fm})$ & $V_{0}(\mathrm{MeV})$ & $r_{0}(\mathrm{fm})$ \\
\hline${ }^{28} \mathrm{Si}+{ }^{120} \mathrm{Sn}$ & 85.89 & 11.04 & 4.35 & 0.667 & 250 & 1.07 \\
${ }^{16} \mathrm{O}+{ }^{120} \mathrm{Sn}$ & 50.41 & 10.73 & 4.37 & 0.648 & 167 & 1.10 \\
\hline \hline
\end{tabular}




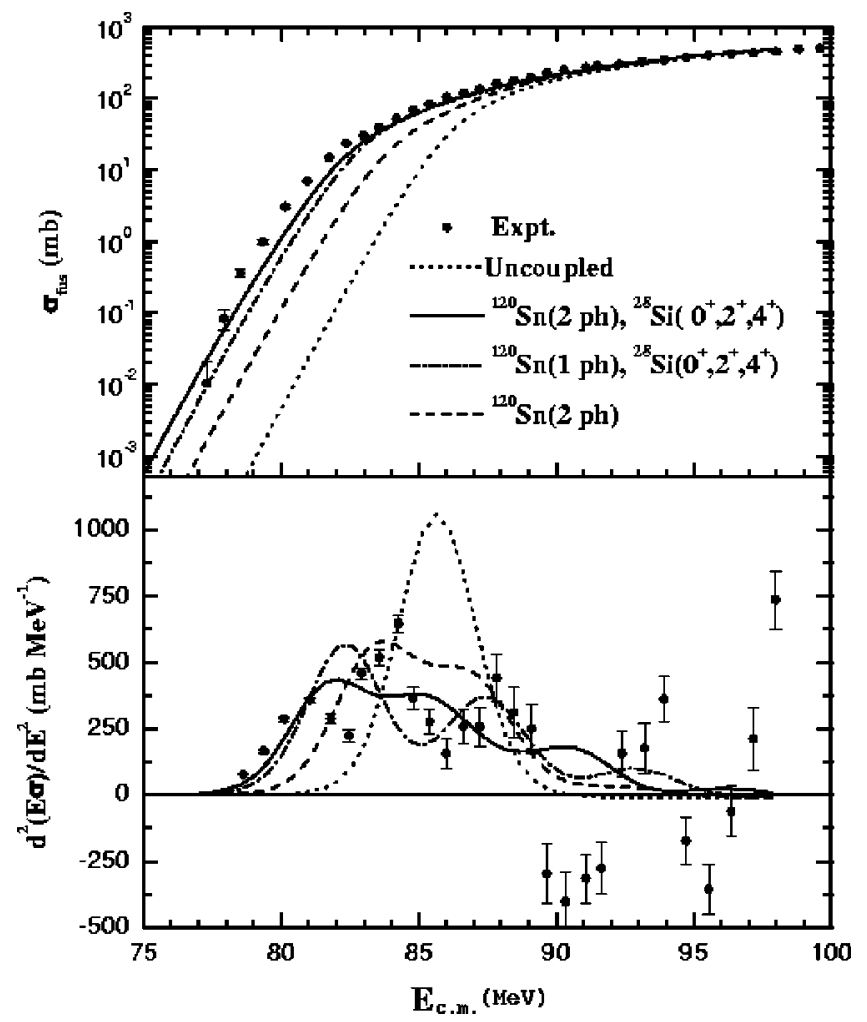

FIG. 4. Fusion excitation function and barrier distribution for ${ }^{28} \mathrm{Si}+{ }^{120} \mathrm{Sn}$ with the results of the exact coupled-channel calculations. For the target nucleus, phonons included are $2^{+}$and $3^{-}$. The rotational states of ${ }^{28} \mathrm{Si}$ coupled are the $0^{+}, 2^{+}, 4^{+}$states. The dashdotted line represents the coupling of rotational states of $\mathrm{Si}$ along with the one-phonon states of Sn whereas the solid line shows the additional coupling to two-phonon states. Dashed line shows the case where ${ }^{28} \mathrm{Si}$ was considered inert.

the second term gives the contribution of Coulomb excitation. The ion-ion potential used in the calculations was a Woods-Saxon parametrization of the Akyüz-Winther potential [16]. The $\beta_{2}, \beta_{3}$ values were taken from the literature $[17,18]$ and are given in Table I. The results of the calculations, which included the $2^{+}, 3^{-}$states of ${ }^{120} \mathrm{Sn}$, are shown in Fig. 2. Excitations in ${ }^{16} \mathrm{O}$ were not included in the calculations, since its excitations only lead to a renormalization of the bare potential due to its high-lying character $[19,20]$. The nuclear potential was adjusted slightly so that the position of the barrier matches with the measured distribution. In calculating the second derivative the energy step used was same as that of the experimental distribution. Although these calculations reproduce the data reasonably well, there is some discrepancy in the fusion barrier distribution at energies smaller than $49 \mathrm{MeV}$.

Exact coupled-channel calculations were then performed for this system using the code CCFULL [21]. This program includes the couplings to full order and thus does not introduce the expansion of the coupling potential. The isocentrifugal approximation is used in the program where one can replace the angular momentum of the relative motion in each channel by the total angular momentum. The finite excitation energies of the intrinsic motions are taken into account. The

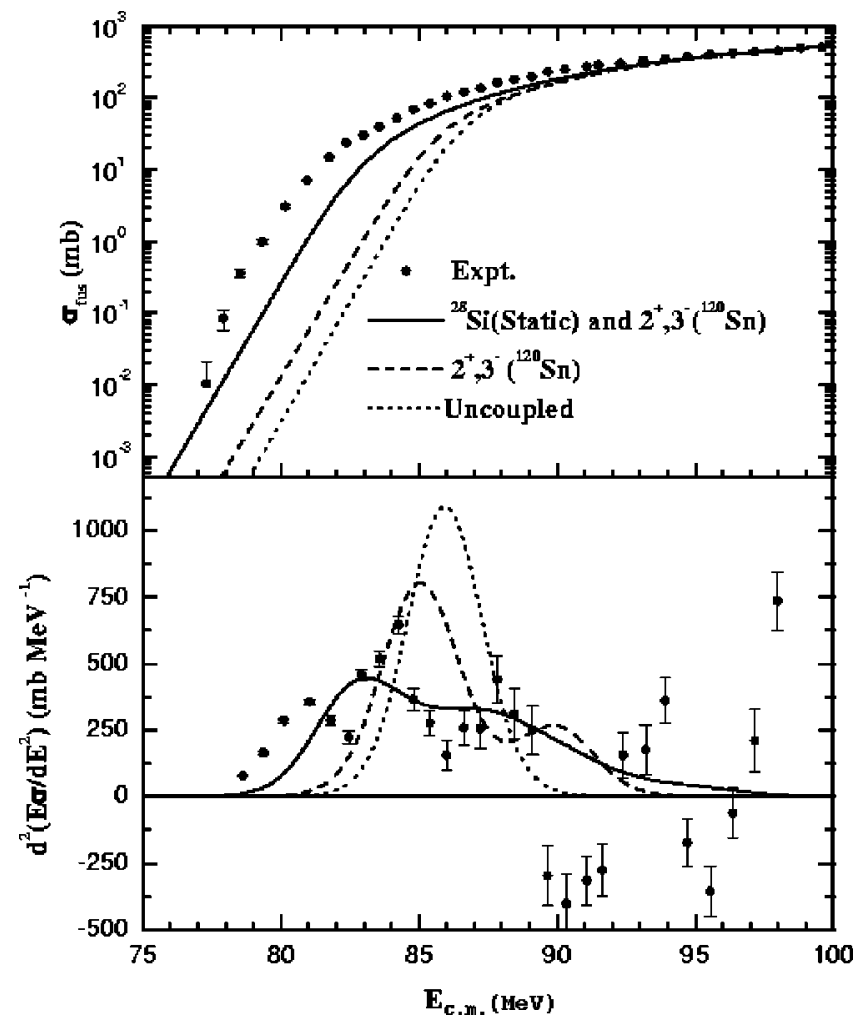

FIG. 5. Fusion excitation function and barrier distribution for ${ }^{28} \mathrm{Si}+{ }^{120} \mathrm{Sn}$. The solid line shows the result of the simplified calculation where $\mathrm{Si}$ was considered as frozen, including coupling to the $2^{+}, 3^{-}$states of $\mathrm{Sn}$. The dashed line is for the case where only the vibrational states of Sn were considered.

program uses the ingoing wave boundary condition inside the Coulomb barrier. The nuclear form factor used is a deformed Woods-Saxon form factor, which includes all order coupling. Coulomb excitation is also included in the coupling matrix element. The relevant parameters of the potential used in the calculation are given in Table II. These parameters were adjusted to nearly match the potential parameters used in the simplified calculation.

The results of the exact calculations are shown in Fig. 3. The dashed line in Fig. 3 is the CCFULL calculation which includes the one-phonon $2^{+}, 3^{-}$states of ${ }^{120} \mathrm{Sn}$. It gives an improved agreement with the data. We found that the calculation including coupling up to two-phonon states of ${ }^{120} \mathrm{Sn}$ gives a good fit to both the barrier distribution and the excitation function. The results are shown by the solid line in Fig. 3. The states included in these calculations were $\left(2^{+}, 3^{-}, 2^{+} \otimes 2^{+}, 2^{+} \otimes 3^{-}\right.$, and $\left.3^{-} \otimes 3^{-}\right)$. Further inclusion of three-phonon states did not significantly alter the calculated barrier distribution (Fig. 3). From these results it is clear that the principal enhancement mechanism in this system is the coupling to the double-phonon states of ${ }^{120} \mathrm{Sn}$. In the calculations including two- or three-phonon states in Sn, a pure vibrational model was assumed and hence the energy of these states was taken to be twice or thrice the energy of the one-phonon state.

Using the coupling scheme established for the above re- 


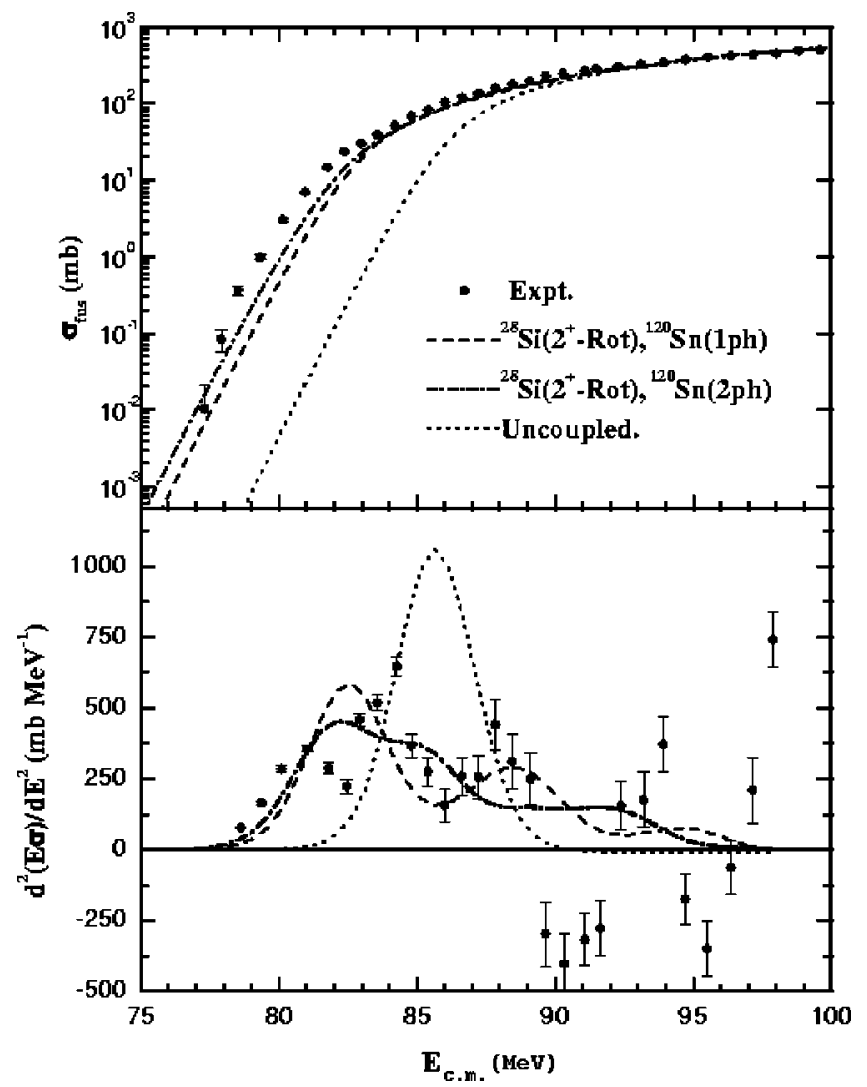

FIG. 6. Fusion excitation function and barrier distribution for ${ }^{28} \mathrm{Si}+{ }^{120} \mathrm{Sn}$. Results of the exact calculations where the vibrational states of the target up to one-phonon (dashed line) and two-phonon (dash-dotted line) states were included along with the $0^{+}, 2^{+}$rotational states of ${ }^{28} \mathrm{Si}$.

action, we next analyzed the ${ }^{28} \mathrm{Si}+{ }^{120} \mathrm{Sn}$ system. We first assumed ${ }^{28} \mathrm{Si}$ to be inert. The calculation was performed using the code CCFULL and the single- and double-phonon states in ${ }^{120} \mathrm{Sn}$ were included. The result of such a calculation underpredicts the cross section by a large amount and the shape of the barrier distribution is not in agreement with the measured distribution (Fig. 4). This clearly shows that there exists a strong enhancement mechanism other than coupling to states of ${ }^{120} \mathrm{Sn}$ and points towards the need to perform calculations including ${ }^{28} \mathrm{Si}$. Since silicon shows rotational structure [22], the rotational states of silicon were coupled in the next calculations. But the way of treating a deformed nuclei in the frozen approximation may not hold in the case of silicon due to the following reasons. There are two conditions to be satisfied for the static or frozen approximation to be valid. The first condition is the assumption of an infinite number of rotational states. So if there is truncation in the rotational band, the static approximation may break down. Second, is the assumption of zero excitation energy for the rotational levels. It has been shown [23,24] that the coupled-channel effect corresponding to coupling to rotational states from $I=0$ to $I=2 N-2$, ignoring the excitation energies, is equivalent to doing a weighted average of the amplitudes corresponding to $N$ orientations of the deformed nuclei. Thus,

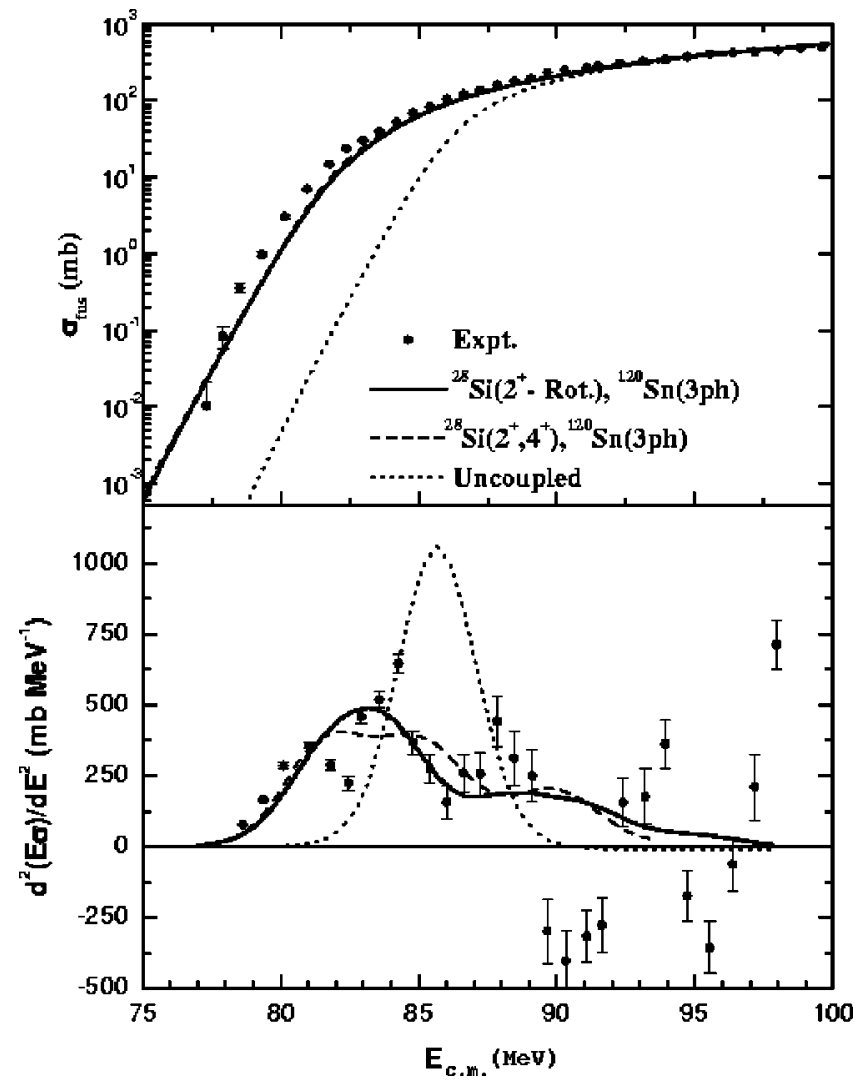

FIG. 7. Fusion excitation function and barrier distribution for ${ }^{28} \mathrm{Si}+{ }^{120} \mathrm{Sn}$. The results of the exact coupled-channel calculations are shown. The solid line shows the result of coupling to vibrational states up to the three-phonon level of ${ }^{120} \mathrm{Sn}$ along with the $0^{+}, 2^{+}$ rotational states of ${ }^{28} \mathrm{Si}$. The dashed line is the result obtained when the $4^{+}$state of ${ }^{28} \mathrm{Si}$ was also included along with the above coupling.

$$
\sigma_{f u s}^{t o t}=\int_{0}^{\pi / 2} \sin \theta \sigma_{f u s}(\theta) d \theta,
$$

where the angle $\theta$ is defined between the symmetry axis of the deformed nucleus and the relative motion. The frozen approximation, which is employed in the CCDEF code assumes an infinite rotational band and zero excitation energy, is a good approximation in the case of heavy deformed nuclei where the number of levels in the ground state rotational band is fairly large and the excitation energies are rather low. In the case of ${ }^{28} \mathrm{Si}$, neither of the conditions is valid as the rotational band is truncated and also the excitation energy of the first $2^{+}$state is too large to be neglected. The frozen approximation is expected to get worse in light nuclei such as ${ }^{28} \mathrm{Si}$ due to the above reasons. Hence we performed exact coupled-channel calculations where the energies of rotational states were explicitly taken into account. For comparison we have also shown the results of simplified calculations (Fig. 5) where silicon was treated as frozen. As evident from the figure, this fails to explain the excitation function and barrier distribution. In the exact calculations, the states coupled were $0^{+}, 2^{+}$, and $4^{+}$of $\mathrm{Si}$ along with the vibrational states 
of Sn (up to two phonons). The results are shown in Fig. 4. It can be seen that the coupling to the rotational states of ${ }^{28} \mathrm{Si}$ brings in an additional enhancement of $2 \mathrm{MeV}$ and gives a reasonable fit to the excitation function. The agreement between the calculated and measured distributions is not, however, very good.

One might wonder whether the truncation of the rotational couplings in ${ }^{28} \mathrm{Si}$ has something to do with this disagreement. It is, however, not the case as can be seen in Fig. 6 . The calculations shown in this figure include only the $2^{+}$ state in ${ }^{28} \mathrm{Si}$, together with the phonon excitations in the target nucleus. The figure shows that the removal of the coupling to the $4^{+}$state does not make any major change. The contribution from the hexadecapole deformation was also estimated by doing calculations with a $\beta_{4}$ value of 0.1 . But this did not seem to make any appreciable change in the excitation function and barrier distribution. We next investigated effects of higher phonon excitations on ${ }^{120} \mathrm{Sn}$. Although they do not play any important role in the ${ }^{16} \mathrm{O}+{ }^{120} \mathrm{Sn}$ reaction, due to the larger $Z_{p} Z_{t}$, the higher phonon excitations might have appreciable strength in the ${ }^{28} \mathrm{Si}+{ }^{120} \mathrm{Sn}$ case. Figure 7 shows results of the CCFULL calculation which includes up to the three-phonon states of ${ }^{120} \mathrm{Sn}$ along with the $2^{+}$state of ${ }^{28} \mathrm{Si}$. The agreement seems to improve significantly. The discrepancy between the experimental data and the calculations around $90 \mathrm{MeV}$ could be due to the reasons given in the previous section. The phonons included in the above calculation were $\left(2^{+}, 3^{-}, 2^{+} \otimes 2^{+}, 2^{+} \otimes 3^{-}, 3^{-} \otimes 3^{-}\right.$, $\left(2^{+}\right)^{2} \otimes 3^{-},\left(2^{+}\right)^{3},\left(3^{-}\right)^{2} \otimes 2^{+}$, and $\left.\left(3^{-}\right)^{3}\right)$. At the same time we performed calculations including couplings to the $2^{+}, 4^{+}$states of ${ }^{28} \mathrm{Si}$ along with coupling to the three-phonon states of ${ }^{120} \mathrm{Sn}$. We found that they do not lead to a good fit to the barrier distribution even though the enhancements produced by both coupling schemes were almost the same.

\section{CONCLUSION}

We performed fusion measurements on the systems ${ }^{16} \mathrm{O}$ and ${ }^{28} \mathrm{Si}+{ }^{120} \mathrm{Sn}$ to identify the channels which are relevant in the reaction process. Analysis of our data for the $\mathrm{O}+\mathrm{Sn}$ system indicates that coupling to the vibrational state of $\mathrm{Sn}$ (up to the two-phonon level) is sufficient to explain the structure of the barrier distribution and the excitation function. There was no indication for the need to couple to the vibrational states of oxygen, whereas the analysis of the $\mathrm{Si}+\mathrm{Sn}$ data clearly brings out the significance of rotational behavior of ${ }^{28} \mathrm{Si}$ in the reaction mechanism and also gives insight into the magnitude of the collective excitation. In this system, the coupling to $0^{+}, 2^{+}$rotational states of ${ }^{28} \mathrm{Si}$ along with phonon coupling up to three-phonon level in ${ }^{120} \mathrm{Sn}$ is found to be the relevant channels. The need for coupling to higher phonon states as the $Z_{p} Z_{t}$ increases is evident from these results. The small disagreement in the barrier distribution at the lowest energies could be due to the coupling to positive $Q$-value transfer channels ( $2 n, 3 n$, and $4 n$ pickup) which were not included in the present calculations. However in the case of weak transfer coupling, the net effect will appear only at the tail of the low-energy side of the barrier distribution.

\section{ACKNOWLEDGMENTS}

The authors wish to acknowledge the help from the accelerator staff at Nuclear Science Center, New Delhi for providing a stable beam during the experiment. We would like to acknowledge Prof. G. K. Mehta, Director (NSC) for his encouragement during the course of this work. Help received from Akhil Jhingan during the experiment is acknowledged. Useful discussion with Dr. M. Dasgupta (ANU) is also acknowledged.
[1] N. Rowley, G. R. Satchler, and P. H. Stelson, Phys. Lett. B 254, 25 (1991); N. Rowley, Nucl. Phys. A538, 205c (1992).

[2] J. R. Leigh, M. Dasgupta, D. J. Hinde, J. C. Mein, C. R. Morton, R. C. Lemmon, J. P. Lestone, J. O. Newton, H. Timmers, J. X. Wei, and N. Rowley, Phys. Rev. C 52, 3151 (1995).

[3] A. M. Stefanini, D. Ackermann, L. Corradi, J. H. He, G. Montagnoli, S. Beghini, F. Scarlassara, and G. F. Segato, Phys. Rev. C 52, R1727 (1995); Phys. Rev. Lett. 74, 864 (1995).

[4] C. R. Morton, M. Dasgupta, D. J. Hinde, J. R. Leigh, R. C. Lemmone, J. P. Lestone, J. C. Mein, J. O. Newton, H. Timmers, N. Rowley, and A. T. Kruppa, Phys. Rev. Lett. 72, 4074 (1994).

[5] J. X. Wei, J. R. Leigh, D. J. Hinde, J. O. Newton, R. C. Lemmon, S. Elfstrom, J. X. Chen, and N. Rowley, Phys. Rev. Lett. 67, 3368 (1991).

[6] J. D. Bierman, P. Chan, J. F. Liang, M. P. Kelly, A. A. Sonzogni, and R. Vandenbosch, Phys. Rev. Lett. 76, 1587 (1996).

[7] M. Dasgupta, D. J. Hinde, J. R. Leigh, R. C. Lemmon, J. C. Mein, C. R. Morton, and H. Timmers, in Proceedings of the
International Workshop on "Heavy-Ion Fusion: Exploring the Variety of Nuclear Properties,' Padua, Italy, 1994, edited by A. M. Stefanini et al. (World Scientific, Singapore, 1994), p. 115.

[8] A. K. Sinha, N. Madhavan, J. J. Das, P. Sugathan, D. O. Kataria, A. P. Patro, and G. K. Mehta, Nucl. Instrum. Methods Phys. Res. A 339, 543 (1994).

[9] A. M. Vinodkumar, K. M. Varier, N. V. S. V. Prasad, D. L. Sastry, A. K. Sinha, N. Madhavan, P. Sugathan, D. O. Kataria, and J. J. Das, Phys. Rev. C 53, 803 (1996); A. M. Vinodkumar, Ph.D. thesis, 1996, Calicut University, India.

[10] N. V. S. V. Prasad, A. M. Vinodkumar, A. K. Sinha, K. M. Varier, D. L. Sastry, N. Madhavan, P. Sugathan, D. O. Kataria, and J. J. Das, Nucl. Phys. A603, 176 (1996).

[11] A. Gavron, Phys. Rev. C 21, 230 (1980).

[12] C. H. Dasso and S. Landowne, Comput. Phys. Commun. 46, 187 (1987); J. Fernandez-Niello and C. H. Dasso, ibid. 54, 409 (1989).

[13] C. H. Dasso, S. Landowne, and A. Winther, Nucl. Phys. A405, 381 (1983); A407, 221 (1983).

[14] M. Dasgupta, A. Navin, Y. K. Agarwal, C. V. K. Baba, H. C. 
Jain, M. L. Jhingan, and A. Roy, Nucl. Phys. A539, 351 (1992).

[15] D. L. Hill and J. A. Wheeler, Phys. Rev. 89, 1102 (1953).

[16] O. Akyüz and A. Winther, in Proceedings of the International School of Physics "Enrico Fermi," Course LXXI, 1979, edited by R. A. Broglia, C. H. Dasso, and R. Riccl (NorthHolland, Amsterdam, 1981), p. 492.

[17] S. Raman, C. H. Malarkey, W. T. Milner, C. W. Nestor, and P. H. Stelson, At. Data Nucl. Data Tables 36, 1 (1987).

[18] R. H. Spear, At. Data Nucl. Data Tables 42, 55 (1989).

[19] K. Hagino, N. Takigawa, and A. B. Balantekin, Phys. Rev. C
56, 2104 (1997).

[20] K. Hagino, N. Takigawa, M. Dasgupta, D. J. Hinde, and J. R. Leigh, Phys. Rev. Lett. 79, 3014 (1997).

[21] K. Hagino, N. Rowley, and A. T. Kruppa, Comput. Phys. Commun. 123, 143 (1999).

[22] M. A. Meyer, I. Venter, and D. Reitmann, Nucl. Phys. A250, 235 (1975).

[23] M. A. Nagarajan, A. B. Balantekin, and N. Takigawa, Phys. Rev. C 34, 894 (1986).

[24] M. V. Andres, X Gomez-Camacho, and M. A. Nagarajan, Phys. Rev. C 45, 1339 (1992). 